\title{
A randomized trial comparing seven-day ranitidine bismuth citrate and clarithromycin dual therapy to seven-day omeprazole, clarithromycin and amoxicillin triple therapy for the eradication of Helicobacter pylori
}

\author{
Sander Veldhuyzen van Zanten $\mathrm{MD}^{1}$, Naoki Chiba $\mathrm{MD}^{2}$, Alan Barkun $\mathrm{MD}^{3}$, Carlo Fallone $\mathrm{MD}^{3}$, \\ Alain Farley $\mathrm{MD}^{4}$, Alan Cockeram MD${ }^{5}$, Chrystian Dallaire $\mathrm{MD}^{6}$, Lorinda Simms $\mathrm{MSc}^{7}$, Barbara Nicholls $\mathrm{BSc}^{7}$
}

S Veldhuyzen van Zanten, N Chiba, A Barkun, et al. A randomized trial comparing seven-day ranitidine bismuth citrate and clarithromycin dual therapy to seven-day omeprazole, clarithromycin and amoxicillin triple therapy for the eradication of Helicobacter pylori. Can J Gastroenterol 2003;17(9):533-538.

OBJECTIVE: To assess Helicobacter pylori eradication after one week dual ranitidine bismuth citrate-clarithromycin (RBC-C) or triple omeprazole, clarithromycin and amoxicillin (OCA) therapy.

METHODS: In this multicentre Canadian trial, $\mathrm{H}$ pylori-positive patients with functional dyspepsia or inactive peptic ulcer disease were randomized to open-label treatment with RBC-C (ranitidine bismuth citrate $400 \mathrm{mg}$ plus clarithromycin $500 \mathrm{mg}$ ) or OCA (omezaprole $20 \mathrm{mg}$, clarithromycin $500 \mathrm{mg}$ and amoxicillin $1000 \mathrm{mg}$ ), given twice a day for seven days. Treatment allocation was randomly assigned. $H$ pylori infection was confirmed by positive ${ }^{13} \mathrm{C}$-urea breath test $\left({ }^{13} \mathrm{C}\right.$-UBT $) . H$ pylori status was reassessed by UBT at least four and 12 weeks after treatment (negative: $\delta^{13} \mathrm{CO}_{2}$ below 3.5 per mil). Intention-to-treat (ITT) eradication rates were determined for all patients with confirmed $\mathrm{H}$ pylori infection. Per protocol (PP) rate was determined for all patients treated with at least two evaluable follow-up visits.

RESULTS: Three hundred five patients were included in the ITT and 222 in the PP analysis. The ITT eradication rates were $66 \%$ for RBC-C and 78\% for OCA. The PP success rates were $84 \%$ for RBC-C and $96 \%$ for OCA. The difference for both ITT 12\% (95\% CI 2 to 22 ) and PP $12 \%$ (95\% CI 4 to 19) were statistically significant, $\mathrm{P}=0.030$ and $\mathrm{P}=0.007$, respectively. Treatment was generally well tolerated.

CONCLUSION: The eradication rate for the seven-day dual RBC-C regimen was lower than that for OCA.

Key Words: Amoxicillin; Bismuth; C difficile colitis; Clarithromycin; Dyspepsia; Eradication; Functional dyspepsia; Helicobacter pylori; Omeprazole; Patient satisfaction; Peptic ulcer disease; Randomized clinical trial; Ranitidine bismuth citrate; Treatment; Treatment failure
Un essai randomisé comparant un traitement à double modalité de sept jours de citrate de bismuth de ranitidine à une thrithérapie de sept jours d'oméprazole, de clarithromycine et d'amoxicilline pour éradiquer une infection Helicobacter pylori

OBJECTIF : Évaluer l'éradication d'une infection Helicobacter pylori après un traitement à double modalité d'un semaine de citrate de bismuth de ranitidine-clarithromycine (RBC-C) ou une thrithérapie d'omézaprole, de clarithromycine et d'amoxicilline (OCA).

MÉTHODES : Au cours d'un essai multicentrique canadien, on a administré de façon aléatoire à des patients $H$ pylori-positifs souffrant d'une dyspepsie fonctionnelle ou d'un ulcère peptique inactif un traitement avec étiquetage en clair de RBC-C (citrate de bismuth de ranitidine $400 \mathrm{mg}$ plus clarithromycine $500 \mathrm{mg}$ ) ou d'OCA (omézaprole $20 \mathrm{mg}$, clarithromycine $500 \mathrm{mg}$ et amoxicilline $1000 \mathrm{mg}$ ), deux fois par jour pendant sept jours. On a sélectionné les patients recevant le traitement au hasard. L'infection $H$ pylori a été confirmée par une épreuve respiratoire à l'urée ${ }^{13} \mathrm{C}\left(\mathrm{ERU}-{ }^{13} \mathrm{C}\right)$. On a réévalué l'état de l'infection $H$ pylori à l'aide d'une ERU au moins quatre à 12 semaines après le traitement (négatif : $\delta^{13} \mathrm{CO} 2$ sous 3,5 par mil). On a déterminé les taux d'éradication selon l'intention de traiter (ITT) chez tous les patients souffrant d'une infection $\mathrm{H}$ pylori confirmée. On a déterminé le taux par protocole (PP) chez tous les patients traités avec au moins deux visites de suivi évaluables.

RÉSULTATS : 305 patients faisaient partie de l'analyse ITT et 222 de l'analyse PP. Les taux d'éradication de l'analyse ITT s'établissaient à $66 \%$ pour le traitement au RBC-C et à $78 \%$ pour le traitement au OCA. Les taux de succès de l'analyse PP s'établissaient à $84 \%$ pour le traitement au RBC-C et à 96\% pour le traitement au OCA. L'écart tant pour l'analyse ITT $12 \%$ (95\% CI 2 à 22) et l'analyse PP 12\% (95 \% CI 4 à 19) était statistiquement important, $\mathrm{P}=0,030$ et $\mathrm{P}=0,007$, respectivement. En général, les patients ont bien toléré le traitement.

CONCLUSION : Le taux d'éradication du traitement à double modalité de sept jours au RBC-C s'est avéré inférieur au OCA.

${ }^{1}$ Dalhousie University, Department of Medicine, Division of Gastroenterology, Halifax, Nova Scotia; ${ }^{2}$ McMaster University, Hamilton, Ontario; ${ }^{3} \mathrm{McGill}$ University Health Centre, Montreal, Quebec; ${ }^{4}$ Centre de Gastro-enterologie de Montreal, Montreal, Quebec; ${ }^{5}$ Saint John Regional Hospital, Saint John, New Brunswick; ${ }^{6}$ Hopital Saint Francois d'Assise, Quebec City, Quebec; 7 GlaxoSmithKline Beecham (Canada) Inc, Mississauga, Ontario

Correspondence: Dr Sander Veldhuyzen van Zanten, Dalhousie University, Department of Medicine, Division of Gastroenterology,

1278 Tower Road, Queen Elizabeth II Health Sciences Centre, Halifax, Nova Scotia B3H 2Y9. Telephone 902-473-1499,

fax902-473-4406, e-mail Zanten@dal.ca

Received for publication May 8, 2003. Accepted May 14, 2003 
$\mathrm{T}$ here is increasing consensus that all patients known to be infected with Helicobacter pylori should be offered treatment $(1,2)$. The target success rate for acceptable therapies to cure Helicobacter infection has been set at $80 \%$ or higher, based on intention-to-treat analysis of randomized controlled trials. Apart from high efficacy, treatments should be simple, well tolerated and safe. Ranitidine bismuth citrate ([RBC], Pylorid, Glaxo Wellcome Inc, Canada) is the first approved drug specifically developed for the treatment of $H$ pylori infection (3). Over the last five years the most successful treatments have been either triple therapy consisting of a proton pump inhibitor (PPI) and clarithromycin with either amoxicillin or metronidazole (PPI-CA or PPI-CM) or quadruple therapy consisting of a PPI with a bismuth compound, metronidazole and tetracycline $(1,2)$. Dual therapy with RBC and clarithromycin (RBC-C) can achieve cure rates of $H$ pylori infection in the range of $70 \%$ to $96 \%(4,5)$. However, this success is achieved with 14-day therapy while triple therapies are successful in seven days. The longer duration of RBC-C therapy makes it more expensive and less convenient. However, because the $\mathrm{RBC}-\mathrm{C}$ regimen uses only two drugs, it is simpler and potentially better tolerated than triple therapies. One study showed that the duration of RBC-C therapy could be decreased to seven days without loss of efficacy (6).

The objective of the current study was to compare the efficacy and safety of seven-day dual therapy of RBC-C to sevenday triple therapy with omeprazole, clarithromycin and amoxicillin (OCA). Patient satisfaction with treatment was also assessed.

\section{METHODS}

This was a computer randomized open-label study carried out in 18 gastroenterology centres and one general practitioner/family practitioner centre across Canada. Patients suffering from chronic dyspepsia with or without proven previous peptic ulcer disease who underwent upper gastrointestinal endoscopy were eligible for the study if they were $H$ pylori positive. Presence of $H$ pylori was determined by a rapid urease test. Patients needed to have confirmation of infection with $H$ pylori by a ${ }^{13} \mathrm{C}$-urea breath test $\left({ }^{13} \mathrm{C}-\mathrm{UBT}\right)$ to be enrolled in the study.

The following were the exclusion criteria: presence of an active duodenal or gastric ulcer observed on endoscopy, history of gastroesophageal reflux disease or endoscopic esophagitis that required ongoing treatment with acid suppressive therapy, renal insufficiency, serious comorbidity precluding participation in the study, or known allergy to any of the drugs used in the study. The use of antibiotics or bismuth-containing medications were not allowed in the four weeks before enrollment. The use of nonsteroidal antiinflammatory drugs was not allowed in the study, but acetylsalicylic acid up to $325 \mathrm{mg}$ a day could be taken. Patients who were taking acid suppressive therapy had to be off $\mathrm{H}_{2}$-blockers or PPIs for at least four weeks before entry into the study. Patients were allowed to have up to one previous attempt at curing $H$ pylori infection. The protocol was approved by the research ethics board of each participating centre. Informed written consent was obtained from each patient.

\section{Treatments}

Patients were randomized to receive either dual therapy with RBC-C (RBC $400 \mathrm{mg}$ twice a day and clarithromycin $500 \mathrm{mg}$ twice a day) or OCA triple therapy consisting of omeprazole $20 \mathrm{mg}$, clarithromycin $500 \mathrm{mg}$ and amoxicillin $1000 \mathrm{mg}$, given twice daily for seven days. Assignment of treatments was randomized using tables of random numbers. Investigators and research personnel were blinded until treatment allocation was assigned. Once patients were assigned their treatment, the medications were provided in an unblinded fashion. Treatment started within 10 days of the endoscopy and ${ }^{13} \mathrm{C}$-UBT. Compliance was assessed by history and pill count of returned medication. A patient was considered compliant if the patient took $80 \%$ of all prescribed medications.

\section{Assessment of $\mathrm{H}$ pylori status}

At endoscopy, biopsies were taken from the antrum and the corpus, and tested by a rapid urease test for $H$ pylori (CLOtest, TriMed Specialties Inc, USA). Positive results were confirmed by a positive ${ }^{13} \mathrm{C}$-UBT (PYLORITEST, Isodiagnostika Inc, Canada) before enrollment in the study. This test has previously been validated (7). The test was considered positive for $\mathrm{H}$ pylori if excess ${ }^{13} \mathrm{CO}_{2}$ per mil was 3.5 or higher. ${ }^{13} \mathrm{C}$-UBTs were performed at least four to six weeks and 12 to 14 weeks after the completion of the seven-day treatment.

Patients were considered negative for $H$ pylori infection only if both follow-up ${ }^{13} \mathrm{C}$-UBTs were negative and evaluable.

\section{Other assessments}

Specific patient symptoms were assessed using a checklist. Patients were seen at the end of the treatment phase to document side effects. A patient satisfaction questionnaire measuring five treatment attributes was developed for this study by GlaxoSmithKline. The instrument has not been previously validated. It was completed by the patient before and after the one-week treatment period.

\section{Study populations}

The safety population was defined as any patient who was randomized and had received at least one dose of study medication. The intent-to-treat (ITT) population was defined as any randomized subject with confirmed $\mathrm{H}$ pylori infection (results of both UBT and CLOtest positive). Patients with discordant UBT results at the week 4 and week 12 follow-up were considered treatment failures in the ITT analysis. The per-protocol (PP) population was defined as subsets of the ITT population. Subjects in the PP population were required to be compliant with study medication, return for follow-up evaluations within the allotted timeframe and not receive concurrent medication known to interfere with the assessment of $H$ pylori status, such as antibiotics.

For the PP analysis, patients were excluded if the week 4 or week 12 evaluation was missing or unavailable, occurred less than 26 days (week 4) or less than 77 days (Week 12) after the end of treatment, or was discordant with either the four- or 12-week evaluation.

\section{Statistical methods}

The primary outcome measure was the $H$ pylori infection eradication rate in the ITT population. The proportion of patients with $H$ pylori eradication in each treatment group was estimated along with the $95 \%$ CI for each proportion. The difference in the proportion of eradication success between treatment groups was reported, including the exact $95 \% \mathrm{CI}$ for the difference in proportions (8). Treatment groups were also compared using a Fisher's Exact Test, appropriate for a two-by-two contingency table. An identical analysis strategy was adopted for the analysis of the eradication rates in the PP population, considered a secondary outcome measure in this study. 
Another secondary outcome, patient satisfaction, was assessed by the patient before and after the one-week treatment period. The pretreatment questionnaire measured how important the attributes were in general on a five-point scale from "not at all important" to "extremely important". The post-treatment questionnaire measured how important the attributes were in relation to the randomized treatment regimen that they had just completed on a five-point scale from "very dissatisfied" to "very satisfied". Post-treatment responses were analyzed by collapsing the five-point scale into a binary outcome consisting of either "satisfied" (very satisfied or somewhat satisfied) or "not satisfied" (neutral or somewhat dissatisfied or very dissatisfied) and then compared using a $\chi^{2}$ test for binary outcomes $(9,10)$. The analysis was done initially based on all patients and then repeated based on the subset of patients who attached some importance (pretreatment) to the given attribute.

\section{Sample size}

Based on the assumption that the ITT eradication rates would be at least $85 \%(1,11,12)$, a sample size of 150 patients per treatment group was required to ensure that the width of the $95 \% \mathrm{CI}$ for the difference in eradication rates would not exceed 16 percentage points.

\section{RESULTS}

Patients were enrolled from October 1998 until November 1999. Three hundred thirteen patients were enrolled. A summary of the disposition of patients in this study is provided in Table 1. Patient numbers in both groups were very similar. Treatment groups were similar with respect to demographics and baseline characteristics (Table 2).

The ITT population consisted of 305 patients and the PP population, 222 patients. The largest group of patients $(\mathrm{N}=56$, $18 \%$ of randomized patients) excluded from the PP analysis were those with missing or discordant UBT results at week 4 and week 12 or those who had follow-up evaluations outside the target time windows (less than 26 days at week 4 or less than 77 days at week 12). Of these 56 patients, at least one follow-up ${ }^{13} \mathrm{C}$-UBT result was missing in 28 patients $(8.9 \%)$. Both ${ }^{13} \mathrm{C}$-UBT results were missing in 10 patients and only one UBT was available in 18 patients. There were 15 patients (5\% of randomized patients) who had discordant UBT results between their week 4 and week 12 follow-up evaluations. Following repeat tests for those patients with unevaluable UBT results, the number of patients with discordant results was reduced to eight. Patients with discordant results were considered to be treatment failures in the ITT analysis.

The $H$ pylori eradication proportions according to the ITT and PP populations show that the OCA triple regimen was significantly better than RBC-C dual therapy in the ITT and PP groups' analysis (Table 3). The ITT difference between OCA and RBC-C was $12 \%$ (95\% CI $2 \%$ to $22 \%, \mathrm{P}=0.030$ ). For the PP analysis, the difference was $12 \%$ (95\% CI $4 \%$ to $19 \%, \mathrm{P}=0.007)$. Twenty patients $(6 \%)$ had received a prior attempt at eradication. The ITT eradication rates for these patients were 63\% (five of eight) for OCA compared with 25\% (three of 12) for RBC-C.

There was no significant difference in eradication rates between the two treatment groups in patients with a prior ulcer history (OCA $80 \%$ or 47 of 59 , RBC-C $65 \%$ or 34 of 52 ), based on the ITT population ( $95 \%$ CI $2 \%$ to $31 \%, \mathrm{P}=0.133$, Fisher's Exact Test). Similar results were noted in the comple-

\begin{tabular}{|c|c|c|c|}
\hline & OCA (n) & RBC-C (n) & Total $(n)$ \\
\hline Enrolled sample size & 157 & 156 & 313 \\
\hline Received no study medication & 1 & 0 & 1 \\
\hline Safety population & 156 & 156 & 312 \\
\hline Negative breath test at baseline & 4 & 3 & 7 \\
\hline Intent-to-treat population & 152 & 153 & 305 \\
\hline Withdrawn due to adverse drug reactio & on 1 & 3 & 4 \\
\hline Consent withdrawn & 2 & 0 & 2 \\
\hline Lost to follow-up & 6 & 3 & 9 \\
\hline Withdrawn due to protocol violation & 1 & 1 & 2 \\
\hline Withdrawn for other reasons & 5 & 5 & 10 \\
\hline UBT not done or unavailable & 27 & 29 & 56 \\
\hline PP population & 110 & 112 & 222 \\
\hline
\end{tabular}

OCA Omeprazole, clarithromycin and amoxicillin; RBC-C Ranitidine bismuth citrate-clarithromycin; PP Per protocol; UBT Urea breath test

\begin{tabular}{lcc}
$\begin{array}{l}\text { TABLE } 2 \\
\text { Demographic data }\end{array}$ & \\
\hline Intent-to-treat population & OCA (n=152) & RBC-C (n=153) \\
\hline Age (years): & $52(13.1)$ & $52(14.1)$ \\
Mean (SD) & $20-81$ & $22-85$ \\
Range & & \\
Sex (n, \%): & $80(53 \%)$ & $79(52 \%)$ \\
$\quad$ Males & $72(47 \%)$ & $74(48 \%)$ \\
Females & & \\
Ethnic origin (n, \%) & $123(81 \%)$ & $135(88 \%)$ \\
White & $8(5 \%)$ & $5(3 \%)$ \\
Black & $17(11 \%)$ & $7(5 \%)$ \\
Asian & $3(2 \%)$ & $5(3 \%)$ \\
American Hispanic & $1(<1 \%)$ & $1(<1 \%)$ \\
Other & & $12(8 \%)$ \\
Previous eradication therapy & $8(5 \%)$ & $141(92 \%)$ \\
Yes & $144(95 \%)$ & \\
No & & $52(34 \%)$ \\
Ulcer history & $59(39 \%)$ & $101(66 \%)$ \\
Yes & $93(61 \%)$ & \\
No &
\end{tabular}

OCA Omeprazole, clarithromycin, amoxicillin; RBC-C Ranitidine bismuth citrate-clarithromycin

ment of ITT patients without prior ulcer history. The eradication rate in all patients with a prior ulcer history was $73 \%$ (81 of 111 ) versus $71 \%$ (138 of 194 patients) without a prior ulcer history, irrespective of treatment group. Other variables such as age and sex were not predictors of treatment success.

As shown in Table 4, patients in the RBC-C treatment group were significantly more satisfied than patients in the OCA treatment group, with respect to the number of drugs that were prescribed ( $82 \%$ versus $69 \%, \mathrm{P}=0.008)$ and the number of pills that they took each day ( $81 \%$ versus $62 \%$, $\mathrm{P}=0.001)$. Conversely, patients in the OCA treatment group were more satisfied with the relief that they received from their study medication than patients in the RBC-C treatment group ( $71 \%$ versus $62 \%, P=0.039$ ). There were no statistically significant treatment differences with the number of side effects experienced while on treatment $(\mathrm{P}>0.35)$.

Overall, compliance with study medication was high. Approximately $89 \%$ of patients took 14 doses of all study 
TABLE 3

Helicobacter pylori eradication rates and $95 \%$ confidence intervals for both treatment arms - for intent-to-treat (ITT) and per-protocol (PP) populations

\begin{tabular}{lccc}
\hline Population & $\mathbf{n}$ & RBC-C & OCA \\
\hline ITT & 305 & $66 \%(101 / 153) ;(59 \%, 74 \%)$ & $78 \%(118 / 152) ;(71 \%, 84 \%)$ \\
PP & 222 & $84 \%(94 / 112) ;(77 \%, 91 \%)$ & $96 \%(105 / 110) ;(92 \%, 99 \%)$ \\
\hline
\end{tabular}

OCA Omeprazole, clarithromycin, amoxicillin; RBC-C Ranitidine bismuth citrate-clarithromycin

\begin{tabular}{|c|c|c|c|}
\hline $\begin{array}{l}\text { What was the patient's ranking of their satisfaction with } \\
\text { the following treatment attributes are: }\end{array}$ & OCA $(n=151)^{*}$ & RBC-C ( $n=153)$ & $\mathbf{P} \S$ \\
\hline \multicolumn{4}{|l|}{ Number of drugs or medications that were prescribed for you } \\
\hline Satisfied ${ }^{\dagger}$ & $104(69 \%)$ & $126(82 \%)$ & $P=0.008$ \\
\hline Not satisfied $¥$ & $42(28 \%)$ & $24(16 \%)$ & \\
\hline No data & $5(3 \%)$ & $3(2 \%)$ & \\
\hline \multicolumn{4}{|l|}{ The number of pills that you took each day } \\
\hline Satisfied & $94(62 \%)$ & $124(81 \%)$ & $P=0.001$ \\
\hline Not satisfied & $52(34 \%)$ & $26(17 \%)$ & \\
\hline No data & $5(3 \%)$ & $3(2 \%)$ & \\
\hline \multicolumn{4}{|l|}{ The number of days that the medication was prescribed } \\
\hline Satisfied & $122(81 \%)$ & $131(86 \%)$ & $P=0.358$ \\
\hline Not satisfied & $24(16 \%)$ & $19(12 \%)$ & \\
\hline No data & $5(3 \%)$ & $3(2 \%)$ & \\
\hline \multicolumn{4}{|l|}{ The number of side effects you experienced while on treatment } \\
\hline Satisfied & $83(55 \%)$ & $88(58 \%)$ & $P=0.752$ \\
\hline Not satisfied & $63(42 \%)$ & $62(41 \%)$ & \\
\hline No data & $5(3 \%)$ & $3(2 \%)$ & \\
\hline \multicolumn{4}{|l|}{ The relief that you received from the medication } \\
\hline Satisfied & $108(71 \%)$ & $95(62 \%)$ & $P=0.039$ \\
\hline Not satisfied & $37(25 \%)$ & $55(25 \%)$ & \\
\hline No data & $6(4 \%)$ & $3(2 \%)$ & \\
\hline
\end{tabular}

*One patient did not have any questionnaire data; †SATISFIED Patients who were either VERY or SOMEWHAT SATISFIED; $¥$ NOT SATISFIED Patients who were either NEUTRAL or SOMEWHATIVERY DISSATISFIED; §Cochran-Mantel-Haenszel Statistic (Modified Ridit Scores) - Van-Elterans Test. OCA Omeprazole, clarithromycin, amoxicillin; RBC-C Ranitidine bismuth citrate-clarithromycin

medication over seven consecutive days during the treatment phase. The compliance rate in the RBC-C treatment group was $95 \%$ versus $84 \%$ in the OCA treatment group, a difference of $11 \%$ in favor of RBC-C (95\% CI $4 \%$ to $18 \%, \mathrm{P}<0.05)$.

\section{Side effects}

Both treatments were generally well tolerated. The incidence of adverse events occurring in at least $5 \%$ of patients overall is summarized in Table 5. Most of these side effects were minor and did not lead to discontinuation in the study. Patients randomized to OCA reported a higher frequency of diarrhea (41\%), compared with 29\% for RBC-C. Patients randomized to $\mathrm{RBC}-\mathrm{C}$ reported a high frequency of abnormal stool color $(38 \%)$ versus $4 \%$ for OCA. Taste disturbance was present in $8 \%$ of RBC-C and $12 \%$ of OCA-treated patients.

Four serious side effects occurred during the study. One patient treated with OCA had an acetaminophen overdose in the follow-up phase. Three RBC-C treated patients respectively had angina pectoris, pulmonary edema and pseudomembranous colitis. The report of pseudomembranous colitis was classified by the investigator as having a reasonable possibility that it was caused by the study medication. This patient recovered without serious consequences, as did the other patients. In total, only four patients withdrew from the study because of adverse effects - one randomized to OCA (allergic reaction) and three to RBC-C (angina pectoris, headache, dizzy spells).

\section{DISCUSSION}

Currently, PPI-based triple therapies with clarithromycin and either amoxicillin or metronidazole are the most commonly recommended regimens worldwide $(1,2)$. The recommended duration is seven days in most countries and 10 days in the USA. In the meta-analyses by Unge et al $(11,12)$ the pooled one-week eradication rate for PPI-CA is $85 \%$ and $83 \%$ for PPI-CM. The eradication rate for the dual therapy of RBC-C given for 14 days has been reported in the range of $73 \%$ to $84 \%$ $(5,13,14)$. One study, only reported in abstract form, has shown that decreasing the duration of RBC-C treatment from 14 to seven days does not lead to a loss in efficacy (6). A shorter course of treatment is helpful in improving compliance and reducing treatment costs. Indeed, in this study, patient satisfaction was greater in the RBC-C treatment group than in the OCA treatment group with respect to the number of medications that were prescribed and the number of pills taken each 
day. Both treatments were well tolerated with mostly mild side effects, that seldomly led to discontinuation of therapy.

Despite these potential advantages, RBC-C dual therapy given for seven days was not as effective as OCA triple therapy in this study (ITT 66\% versus 78\%, PP 84\% versus 95\%, respectively). The difference between RBC-C and OCA was statistically significant. We consider the $12 \%$ difference to be clinically meaningful, especially since subsequent studies using RBC-based triple therapy with clarithromycin and either amoxycillin or metronidazole achieve higher cure rates that are equal to PPI-based triple therapy.

Both treatment regimens were not as effective as reported in prior studies. Although more effective than RBC-C, the 78\% eradication rate with OCA was slightly below the ITT target eradication rate of $80 \%$, recommended in several consensus guidelines $(1,2)$. These results cannot be explained by a higher failure rate in patients with previous attempts of eradication, as only 20 patients had such prior attempts and exclusion of these patients did not alter the results. However, the PP results are well above $80 \%$ for both treatments. There was no significant difference in cure rates in patients with or without a history of ulcers in the past, suggesting that this does not influence treatment results. However, this is based on a subgroup analysis. The study was not powered to specifically answer the question of whether a prior ulcer history is a predictor of treatment results.

Triple therapy using a PPI with clarithromycin and amoxicillin has been tested in several clinical trials in Canada. In the DU-MACH Study, seven-day treatment with OCA achieved a cure of H pylori infection ITT 78\%, PP 87\% (15). In a separate Canadian study comparing omeprazole to esomeprazole with clarithromycin and amoxicillin, ITT results were $90 \%$ and $88 \%$, and PP results were both 91\% (16).

One possible explanation for a decrease in cure rates of $H$ pylori infection is clarithromycin resistance. However, the little available data on resistance to clarithromycin in Canada show that the rates are low, from $2 \%$ to $4 \%(17-19)$. This is in contrast to data from the USA where higher rates of clarithromycin resistance of $6 \%$ to $15 \%$ have been reported (2022). Clarithromycin resistance was not assessed in this study. However, it is possible that $H$ pylori resistance to clarithromycin has increased in Canada as a result of more widespread use, and this deserves further study.

Studies have shown that triple therapy of RBC-C with either metronidazole or amoxicillin achieve similar high eradication rates ranging from $80 \%$ to $90 \%$ as the PPI triple therapies (23). Consequently, RBC-C triple therapy appears to have largely replaced the RBC-C dual therapy. Interestingly, in a recent meta-analysis by Gisbert et al (24) it was shown that, when $\mathrm{RBC}$ is given together with clarithromycin and metronidazole, the success rate was slightly higher when compared with PPI triple therapy with clarithromycin and metronidazole. Although most included studies did not provide data on the effect of metronidazole resistance on eradication rates, the study did suggest that in the presence of metronidazole resistance, RBC-C triple therapy is better able to overcome metronidazole resistance than a triple PPI-CM regimen (23). Another systematic review confirmed this finding (25).

In our study, two UBTs were administered four to six and 12 to 14 weeks after the seven-day treatment. UBT has the advantage that it does not require endoscopy, and if ${ }^{13} \mathrm{C}$-urea is used rather than ${ }^{14} \mathrm{C}$-urea, the test can be done outside hospi-

\begin{tabular}{|c|c|c|}
\hline $\begin{array}{l}\text { Safety patient population } \\
(\mathrm{n}=312)\end{array}$ & $\begin{array}{c}\text { OCA } \\
(n=156)\end{array}$ & $\begin{array}{l}\text { RBC-C } \\
(n=156)\end{array}$ \\
\hline Adverse events experienced ${ }^{*}$ & $129(83)$ & $128(82)$ \\
\hline Diarrhea & $64(41)$ & $45(29)$ \\
\hline Abnormal stool colour & $6(4)$ & $59(38)$ \\
\hline Headaches & $26(17)$ & $17(11)$ \\
\hline Nausea and vomiting & $23(15)$ & $16(10)$ \\
\hline Taste disturbance & $18(12)$ & $12(8)$ \\
\hline Abdominal discomfort and pain & $14(9)$ & $14(9)$ \\
\hline Dyspeptic symptoms & $11(7)$ & $13(8)$ \\
\hline Malaise and fatigue & $11(7)$ & $8(5)$ \\
\hline Dizziness & $12(8)$ & $5(3)$ \\
\hline
\end{tabular}

${ }^{*}$ All data expressed as $n(\%)$. OCA Omeprazole, clarithromycin and amoxicillin; RBC-C Ranitidine bismuth citrate-clarithromycin

tals (26). The study protocol required two UBT's done six to eight weeks apart. In this study 15 of 313 patients (4.8\%) had discordant results between follow-up evaluations. This suggests that in future clinical trials, a single UBT, performed at least four weeks after treatment is completed, is sufficient to document cure of the infection.

In this study, patient satisfaction data was collected prestudy and following one week of treatment. As little work had been done in this area, an importance-performance questionnaire was designed with the intent of determining first which factors were important to patients, and second, how the treatments compared with respect to the attributes that were deemed important. The results of the analysis, however, demonstrated that despite the perceived importance of baseline attributes, such as fewer medications and lower number of pills, this did not have any impact on the patient's level of satisfaction after seven days of treatment. Thus, the design and validation of future questionnaires may only need to be performed assessing post-treatment satisfaction. Patients were more satisfied in the RBC-C group with the number of drugs and the number of pills that they were required to take each day, indicating that patients are concerned about taking large amounts of medications. Patients in the OCA group were more satisfied than RBC-C treated patients with the relief that they received from the treatment, indicating a link between symptoms and treatment success.

In conclusion, a seven-day dual therapy regimen of RBC-C was not as effective as a seven-day OCA triple therapy. This suggests that when prescribing RBC, a seven-day RBC-C triple therapy with either amoxicillin or metronidazole would be preferred over RBC-C dual therapy. Unfortunately, due to lack of physician demand, RBC is no longer available in Canada and several other countries.

ACKNOWLEDGEMENTS: This research was sponsored by GlaxoSmithKline (Canada) Incorporated. The authors would like to express their gratitude to the following additional investigators for their participation in the this study: Drs P Campbell, L Cohen, W Depew, D Duerksen, R Hunt, R LaHaie, B Lasko, C Massicotte, P Paré, I Prokopiw, E Shaffer and AH Steinhart. Dr Veldhuyzen van Zanten is the recipient of a Nova Scotia Clinical Research Scholar Award. 


\section{REFERENCES}

1. Malfertheiner P, Megraud F, O'Morain C, et al and the European Helicobacter pylori study Group (EHPSG). Current concepts in the management of Helicobacter pylori infection - The Maastricht 2-2000 Consensus Report. Aliment Pharmacol Ther 2002;12:167-80.

2. Hunt R, Fallone C, Veldhuyzen van Zanten S, Sherman P, Smaill F, Thomson A on behalf of the Canadian Helicobacter study Group. Risks and benefits of Helicobacter pylori eradication: Current status. Can J Gastroenterol 2002;16:57-62.

3. Pylorid product monograph. Glaxo Wellcome Inc. 1998.

4. Pipkin GA, Dixon JS, Williamson R, Wood JR. Clarithromycin dual therapy regimens for eradication of Helicobacter pylori: A review. Helicobacter 1997:2:159-71.

5. Chiba N, Hunt RH, Thomson AB. Ranitidine bismuth citrate. Can J Gastroenterol 2001;15:389-98.

6. Thjodleifsson B, Beker JA, Costa Mira F, Farley A, Mitchell TR, Roberts PM. Ranitidine bismuth citrate with clarithromycin given for 7 days effectively eradicates $H$ pylori. Gastroenterology 1998;114:G1267.

7. Mock T, Yatscoff R, Foster R, et al. Clinical validation of the Helikit: A ${ }^{13} \mathrm{C}$ urea breath test used for the diagnosis of Helicobacter pylori infection. Clin Biochem 1999;32:59-63.

8. Daniel WW. Biostatistics: A Foundation for Analysis in the Health Sciences, 4th ed. New York: Wiley, 1987.

9. Martilla JA, James JC. Importance-performance analysis. J Mark 1997;41:77-9.

10. Lehmann EL. Nonparametrics: Statistical Methods Based on Ranks. San Francisco: Holden-Day Inc, 1975.

11. Unge $P$. What other regimens are under investigation to treat Helicobacter pylori infection? Gastroenterology. 1997;113(Suppl 6):S131-48.

12. Unge P. Antimicrobial treatment of $H$ pylori infection - a pooled efficacy analysis of eradication therapies. Eur J Surg 1998;Suppl(582):16-26.

13. Hopkins RJ. Current FDA-approved treatments for Helicobacter pylori and the FDA approval process. Gastroenterology 1997;113:S126-30.

14. Gisbert JP, Pajares JM, Valle J. Ranitidine bismuth citrate therapy regimens for treatment of Helicobacter pylori infection: A review. Helicobacter 1999;4:58-66.

15 Veldhuyzen van Zanten SJO, Bradette M, Farley A, et al. The DU-MACH Study: Eradication of Helicobacter pylori and ulcer healing in patients with acute duodenal ulcer using omeprazole based triple therapy. Aliment Pharmacol Ther 1999;13:289-95.

16. Veldhuyzen van Zanten SJO, Lauritsen K, Delchier JC, et al. Oneweek triple therapy with esomeprazole provides effective eradication of Helicobacter pylori in duodenal ulcer disease. Aliment Pharmacol Ther 2000;14:1605-11.

17. Best LM, Haldane DJ, Bezanson GS, Veldhuyzen van Zanten SJ. Helicobacter pylori: Primary susceptibility to clarithromycin in vitro in Nova Scotia. Can J Gastroenterol 1997;11:298-300.

18. Fallone CA. Epidemiology of the antibiotic resistance of Helicobacter pylori in Canada. Can J Gastroenterol 2000;14:879-82.

19. Taylor ED, Jiang Q, Fedorak RN. Antibiotic susceptibilities of Helicobacter pylori strains isolated in the Province of Alberta. Can J Gastroenterol 1998.12:295-8.

20. Osato MS, Reddy R, Graham DY. Metronidazole and clarithromycin resistance amongst Helicobacter pylori isolates from a large metropolitan hospital in the United States. Int J Antimicrob Agents 1999;12:341-7.

21. Laine L, Fennerty MB, Osato M, et al. Esomeprazole-based Helicobacter pylori eradication therapy and the effect of antibiotic resistance: Results of three US multicenter, double blind trials. Am J Gastroenterol 2000;95:3393-8.

22. Meyer JM, Silliman NP, Wang W, et al. Risk factors for Helicobacter pylori resistance in the United States: The surveillance of $H$ pylori anti-microbial resistance partnership (SHARP) study, 1993-1999. Ann Intern Med 2002;136:13-24.

23. Laheij RJ, Rossum LG, Jansen JB, Straatman H, Verbeek AL. Evaluation of treatment regimens to cure Helicobacter pylori in meta-analysis. Aliment Pharmacol Ther 1999;13:857-64.

24. Gisbert JP, Gonzalez L, Calvet X, Roque M, Gabriel R, Pajares JM. Helicobacter pylori eradication: Proton pump inhibitor vs. ranitidine bismuth citrate plus two antibiotics for 1 week - A meta-analysis of efficacy. Aliment Pharmacol Ther 2000:14:1141-50.

25. Janssen MJ, Van Oijen AH, Verbeek AL, Jansen JB, De Boer WA. A systematic comparison of triple therapies for treatment of Helicobacter pylori infection with proton pump inhibitor/ranitidine bismuth citrate plus clarithromycin and either amoxicillin or a nitroimidazole. Aliment Pharmacol Ther 2001;15:613-24.

26. Fallone C, Veldhuyzen S, Chiba N. The urea breath test for Helicobacter pylori infection: Taking the wind out of the sails of endoscopy. CMAJ 2000;162:371-2. 


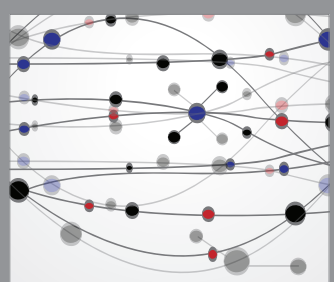

The Scientific World Journal
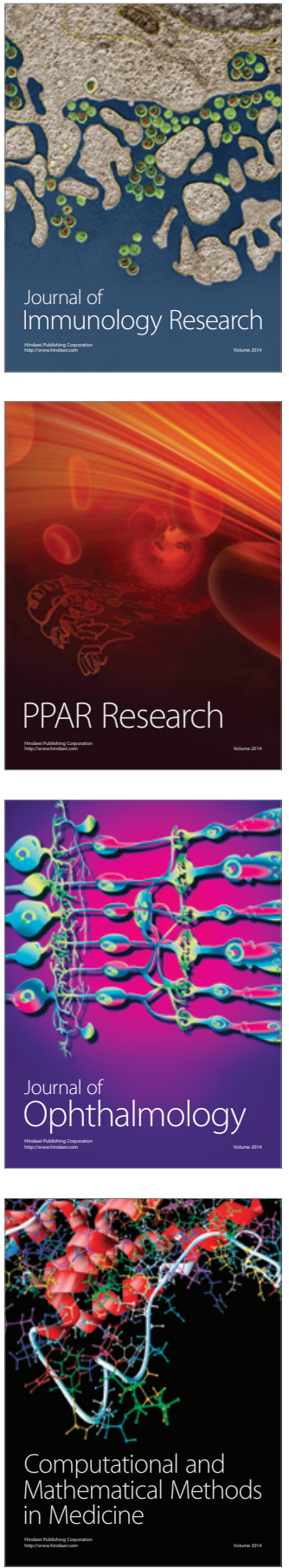

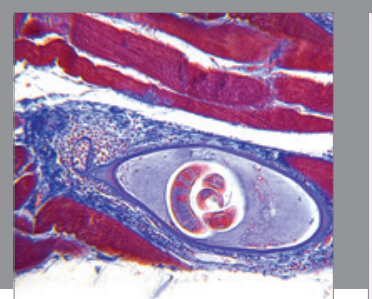

Gastroenterology Research and Practice

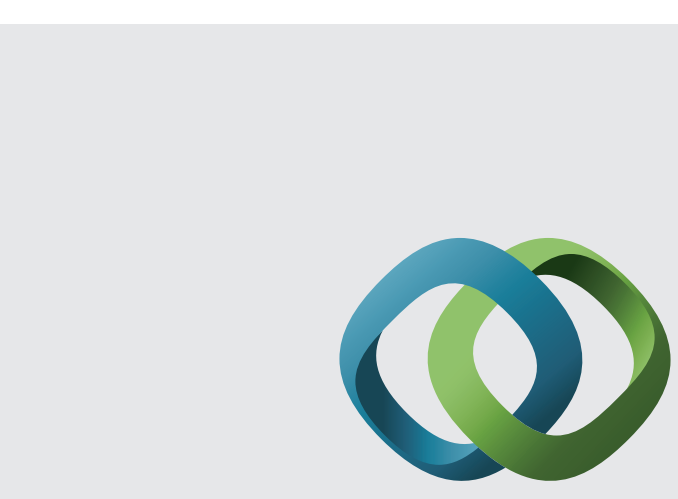

\section{Hindawi}

Submit your manuscripts at

http://www.hindawi.com
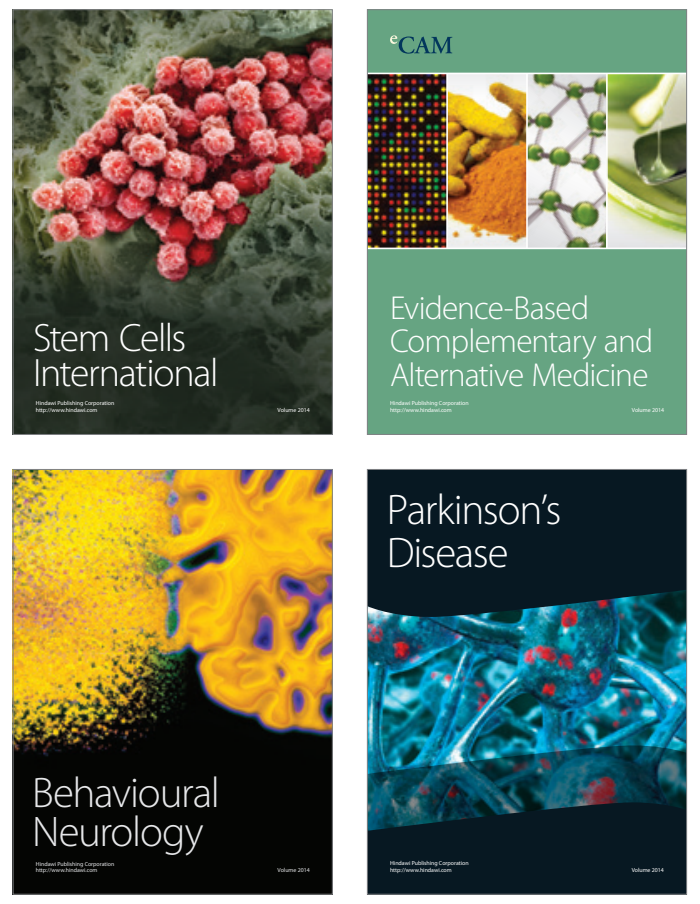
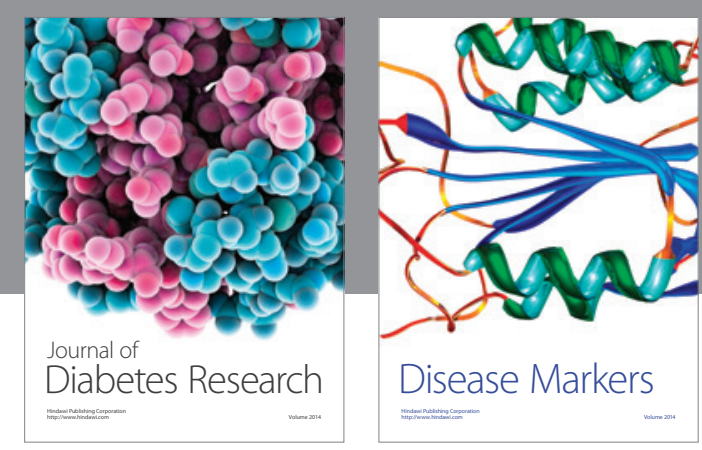

Disease Markers
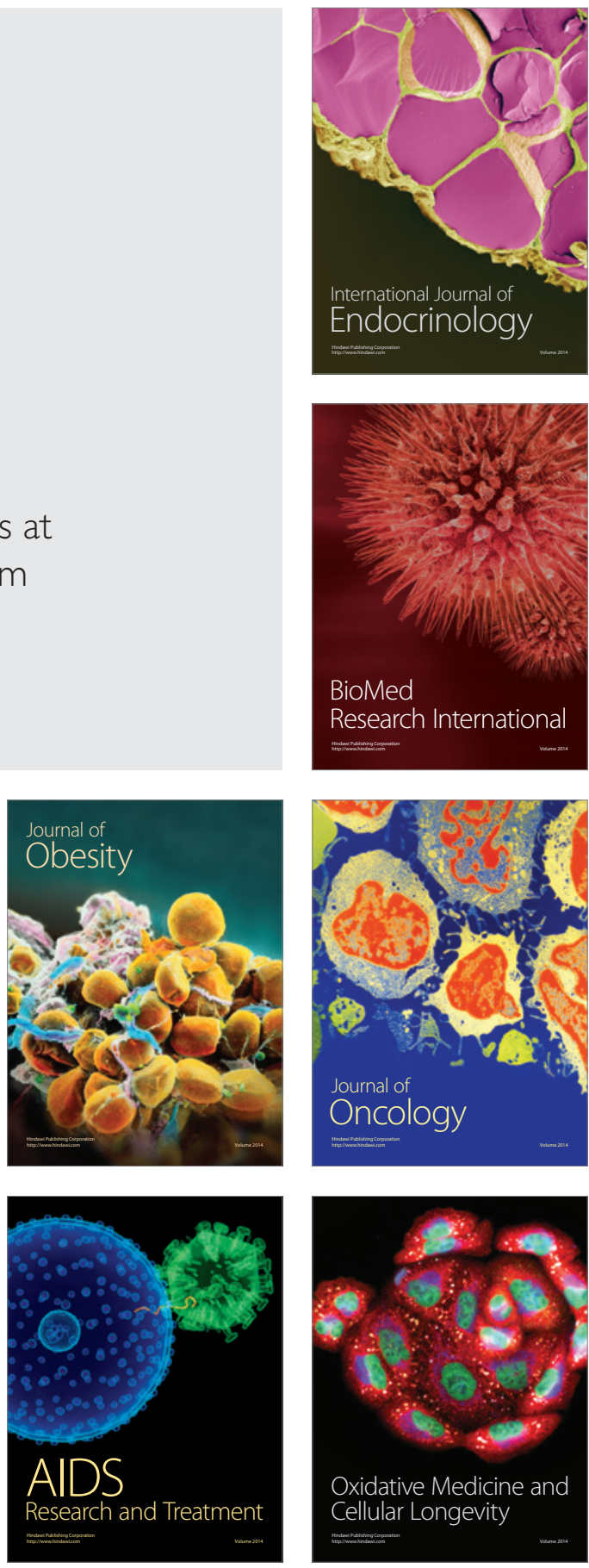